\title{
A general treatment of geometric phases and dynamical invariants
}

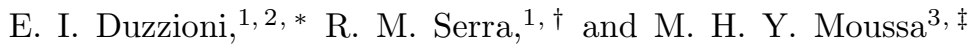 \\ ${ }^{1}$ Centro de Ciências Naturais e Humanas, Universidade Federal do ABC, \\ Rua Santa Adélia 166, Santo André, São Paulo, 09210-170, Brazil \\ ${ }^{2}$ Departamento de Física, Universidade Federal de São Carlos, 13565-905, São Carlos, SP, Brazil \\ ${ }^{3}$ Instituto de Física de São Carlos, Universidade de São Paulo, \\ Caixa Postal 369, São Carlos, São Paulo, 13560-970, Brazil
}

\begin{abstract}
Based only on the parallel transport condition, we present a general method to compute Abelian or non-Abelian geometric phases acquired by the basis states of pure or mixed density operators, which also holds for nonadiabatic and noncyclic evolution. Two interesting features of the nonAbelian geometric phase obtained by our method stand out: i) it is a generalization of Wilczek and Zee's non-Abelian holonomy, in that it describes nonadiabatic evolution where the basis states are parallelly transported between distinct degenerate subspaces, and ii) the non-Abelian character of our geometric phase relies on the transitional evolution of the basis states, even in the nondegenerate case. We apply our formalism to a two-level system evolving nonadiabatically under spontaneous decay to emphasize the non-Abelian nature of the geometric phase induced by the reservoir. We also show, through the generalized invariant theory, that our general approach encompasses previous results in the literature.
\end{abstract}

PACS numbers: 03.65.Ca,03.65.Vf, 03.65.Yz

Introduction. The concept of geometric phase (GP) was transposed to the domain of quantum systems undergoing cyclic adiabatic evolution by M. Berry [1], after having been introduced by Pancharatnam in connection with interference of light waves with distinct polarizations [2]. After Berry's discovery, Aharonov and Anandan [3] removed the need for adiabatic evolution and Samuel and Bhandari [4] extended the concept of GP to noncyclic and nonunitary evolutions, introducing the notion of geodesic closure in the projective Hilbert space. In recent years, the possibility of achieving fault-tolerant quantum computation [5, 6, 7] invoked the controversial subject of GPs for open quantum systems, where the dynamic is generally nonunitary. In this case the GPs have been defined by different methods: using phenomenological approaches [8], stochastic fields [9], the usual master equation [10] and quantum jumps [11] techniques, apart from state purification [12], mean values of distributions 13], interferometric [14], and superoperator methods 15, 16, 17].

Parallel to these advances in the understanding of GPs, the dynamical invariants (DIs) proposed by Lewis and Riesenfeld [18] to handle time-dependent Hamiltonians, have been applied to a number of problems, including recent advances in cavity quantum electrodynamics [19] and Bose-Einstein condensates 20]. Based on the DIs, Morales [21] and Mizrahi 22] introduced, independently, a convenient way to compute the evolution of the GPs, which was used to suggest an interferometric experiment to measure GPs induced by a Stark shift in cavity quantum electrodynamics [23].

In this letter, we rely only on the parallel transport condition to obtain a general formal expression to compute the GPs acquired by the basis states of a density operator under unitary or nonunitary, adiabatic or nona- diabatic, and cyclic or noncyclic evolutions. The DIs applied to the density matrix [24] are required to account for the time-evolution of the GPs in a general scenario of open quantum systems. Apart from showing that our method reproduces previous results in literature, we use it to compute the GP acquired by a two-level system under spontaneous decay. We show unambiguously that this GP turns out to be non-Abelian even in nondegenerate system. Thus, it is clear from this general approach that the non-Abelian character of the GP is wholly associated with the transitional dynamics of the basis states. Moreover, our treatment generalizes Wilczek and Zee's 25] non-Abelian holonomy, in that it describes nonadiabatic evolutions in which the basis states are parallelly transported between distinct degenerate subspaces.

Parallel transport condition. To compute the GPs acquired by the time-dependent states $\{|\lambda, a ; t\rangle\}$ of an orthonormal basis, where $\lambda$ is $g$-fold degenerate $(a=1,2, \ldots, g)$, we first introduce the condition for parallel transport of a state vector, namely that ${ }^{\prime \prime}\left\langle\lambda^{\prime}, a^{\prime} ; t\left|d_{t}\right| \lambda, a ; t\right\rangle_{\|}=0$ [26]. The evolution operator responsible for the parallel transport during the time interval $t,|\lambda, a ; t\rangle_{\|}=V_{\|}(t)|\lambda, a ; 0\rangle$, may be written as $V_{\|}(t)=\sum_{\lambda, \lambda^{\prime}} \sum_{a, a^{\prime}}\left(V_{\|}\right)_{\lambda \lambda^{\prime}}^{a a^{\prime}}(t)|\lambda, a ; t\rangle\left\langle\lambda^{\prime}, a^{\prime} ; 0\right|$. Substituting $V_{11}(t)$ in the parallel transport condition, we find the equation for its coefficients in the matrix form $\dot{V}_{\text {॥ }}(t)=i A(t) V_{\|}(t)$, whose solution is given by

$$
V_{\Perp}(t)=T \exp \left(i \int_{0}^{t} d \tau A(\tau)\right)
$$

where $V_{\|}(0)=1$, the elements of the non-Abelian connection $A(\tau)$ are $A_{\lambda, \lambda^{\prime}}^{a, a^{\prime}}(\tau)=i\left\langle\lambda, a ; \tau\left|d_{\tau}\right| \lambda^{\prime}, a^{\prime} ; \tau\right\rangle$, and $T$ is the time-ordering operator. The unitary matrix $V_{11}(t)(A(\tau)$ being Hermitian) accounts for a cyclic and 
non-Abelian GP. As we are also concerned with noncyclic evolutions, we must account for the relative phase acquired by the parallelly transported state $|\lambda, a ; t\rangle_{\|}$ with respect to its starting point $|\lambda, a ; 0\rangle$, given by arg $\left\{\langle\lambda, a ; 0 \mid \lambda, a ; t\rangle_{\|}\right\}=\arg \left\{\left\langle\lambda, a ; 0\left|W(t, 0) V_{\|}(t)\right| \lambda, a ; 0\right\rangle\right\}$, with the elements of the overlap matrix $W$ reading $W_{\lambda, \lambda^{\prime}}^{a, a^{\prime}}(t, 0)=\left\langle\lambda, a ; 0 \mid \lambda^{\prime}, a^{\prime} ; t\right\rangle$. We note that, to transport a subspace without rotating it locally, it must remain parallel to itself during the time interval from $t$ to $t+\delta t$, i.e., $W_{\lambda, \lambda^{\prime}}^{a, a^{\prime}}(t+\delta t, t)=\left\langle\lambda, a ; t \mid \lambda^{\prime}, a^{\prime} ; t+\delta t\right\rangle \simeq$ $\delta_{\lambda, \lambda^{\prime}} \delta_{a, a^{\prime}}$. Consequently, a necessary and sufficient condition stipulates that $A_{\lambda \lambda^{\prime}}^{a, a^{\prime}}(t)=0$, and hence $A(t)=0$.

As observed in Ref. [27], in the general case, where the overlap matrix $W(t, 0)$ is restricted to an incomplete subspace $\left(\sum_{\lambda, a}|\lambda, a ; t\rangle\langle\lambda, a ; t| \neq \mathbf{1}\right)$, it must be decomposed in the polar form $W=R U, R$ being a positive-definite (Hermitian) matrix (det $R>0)$ and $U$ a unitary matrix $\left(\operatorname{det} U=\mathrm{e}^{i \varphi}\right)$, such that $\mathrm{e}^{i \varphi}=\operatorname{det} W / \operatorname{det} R$. In the case where $R$ is a positive-semidefinite matrix ( $\operatorname{det} R=0$ ), the Moore-Penrose pseudoinverse must be used to evaluate the matrix $U$. Then, the non-Abelian, nonadiabatic and noncyclic GP acquired by the basis states $\{|\lambda, a ; t\rangle\}$ turns out to be

$$
\mathcal{O}(t, 0)=U(t, 0) V_{\Perp}(t),
$$

where $U$ is the unitary part of $W$. Although Eq. (2) has already been published by Mostafazadeh [28] and Kult et al. [27], in both cases the analyzed non-Abelian geometric phases arise from the usual degeneracy, i.e., from transitions inside degenerate subspaces. In a more general scenario, we have extended the analyses in Refs. [27, 28] to cases of transitional dynamics which connect different degenerate and/or nondegenerate subspaces. As discussed below, we found that transitional dynamics, even between nondegenerate states, is the source of a non-Abelian geometric phase. Moreover, when considering the case of transitional dynamics connecting different degenerate subspaces, our analysis reveals a generalization of Wilczek and Zee's non-Abelian holonomy, since it describes nonadiabatic evolutions where the basis states are parallelly transported from one degenerate subspace to another. The definition of parallel transport used here appear similar to that used in Ref. [29], however, our geometric quantity (2) is different from that one defined in [29]. It is important to note that the treatment presented here is general and includes several kinds of system dynamics.

The general expression for $\mathcal{O}(t, 0)$ can be simplified in three particular cases: transitional $(\mathrm{t})$ dynamics in a degenerate $(\mathrm{d})$ subspace $\left(\mathcal{O}^{\mathrm{t}, \mathrm{d}}(t, 0)=\left[U(t, 0) V_{\mathrm{\|}}(t)\right]^{\mathrm{t}, \mathrm{d}}\right)$ and transitional $\left(\mathcal{O}^{\mathrm{t}, \text { nd }}(t, 0)=\left[U(t, 0) V_{\Perp}(t)\right]^{\mathrm{t}, \text {,nd }}\right)$ and nontransitional (nt) $\left(\mathcal{O}^{\text {nt,nd }}(t, 0)=\left[U(t, 0) V_{\Perp}(t)\right]^{\text {nt,nd }}\right) d y-$ namics in a nondegenerate (nd) subspace. The three different cases mentioned above, covering all possible evo- lutions of the basis states, will be explored below in the context of the DIs for a general Lindblad evolution.

Gauge-invariance of the GP. Under the gauge transformation $|\lambda, a ; t\rangle^{\prime}=\sum_{\nu, b} M_{\nu, \lambda}^{b, a}(t)|\nu, b ; t\rangle$, where $M$ is a unitary matrix, the operator $V_{11}(t)$ becomes $V_{\|}^{\prime}(t)=M^{\dagger}(t) V_{\text {॥l }}(t) M(0)$, with $A^{\prime}(t)=M^{\dagger}(t) A(t) M(t)+$ $i M^{\dagger}(t) \dot{M}(t)$. In its turn, $U(t, 0)$ becomes $U^{\prime}(t, 0)=$ $M^{\dagger}(0) U(t, 0) M(t)$. From these transformed expressions, it follows that $\mathcal{O}^{\prime}(t, 0)=M^{\dagger}(0) \mathcal{O}(t, 0) M(0)$, making the trace and the eigenvalues of $\mathcal{O}^{\prime}(t, 0)$ observable gauge invariants.

Summarizing our method, we first choose the timedependent basis to expand the state of the system $\rho(t)$. Next, we analyze the evolution of the basis states to verify if there are transitions or not between them. With these informations we build, through Eq. (2), the operators $\mathcal{O}^{\mathrm{t}, \mathrm{d}}(t, 0)=\left[U(t, 0) V_{\|}(t)\right]^{\mathrm{t}, \mathrm{d}}$, or $\mathcal{O}^{\mathrm{t}, \mathrm{nd}}(t, 0)=$ $\left[U(t, 0) V_{\|}(t)\right]^{\mathrm{t}, \text { nd }}, \quad$ or $\mathcal{O}^{\text {nt }, \text { nd }}(t, 0)=\left[U(t, 0) V_{\|}(t)\right]^{\text {nt,nd }}$, given the geometric phases acquired by the basis states under a general evolution.

$G P$ and the DIs. To illustrate the appearance of the GPs for the different types of evolution discussed above, it is convenient to assume the basis states $\{|\lambda, a ; t\rangle\}$ to be the eigenstates of an Hermitian DI, $I(t)|\lambda, a ; t\rangle=$ $\lambda(t)|\lambda, a ; t\rangle$, since the invariant method is applicable for adiabatic and nonadiabatic processes under unitary and nonunitary evolutions. Moreover, the information about open quantum dynamics is naturally carried through the eigenstates of the invariant operators. In fact, the DIs [18] associate with any density operator $\rho(t)$ the time-conserved quantity $I(t)$, called invariant, which satisfies the condition $\mathrm{d}\langle I(t)\rangle / \mathrm{d} t \equiv 0$ 24]. From the Lindblad form for the density operator of an open quantum system described by the Hamiltonian $H_{0}(t)$, given by $\partial_{t} \rho(t)=-i\left[H_{0}(t), \rho(t)\right]+$ $\sum_{i, j} \gamma_{i j}(t)\left\{\left[\Gamma_{i}, \rho(t) \Gamma_{j}^{\dagger}\right]+\left[\Gamma_{i} \rho(t), \Gamma_{j}^{\dagger}\right]\right\}$, the evolution of the invariant operator following from $\langle I(t)\rangle=$ $\operatorname{Tr}[I(t) \rho(t)]$ becomes

$$
\begin{aligned}
\partial_{t} I(t) & =-i\left[H_{0}(t), I(t)\right]+\sum_{i, j} \gamma_{i j}(t)\left\{\Gamma_{j}^{\dagger}\left[\Gamma_{i}, I(t)\right]\right. \\
& \left.+\left[I(t), \Gamma_{j}^{\dagger}\right] \Gamma_{i}\right\}
\end{aligned}
$$

where $\Gamma_{i}$ are the Lindblad operators coming from the action of an environment and $\gamma_{i j}(t)$ are the coupling strengths. We observe that the Lindblad equation presented above applies for the case where the time scale related to the rate of change of the time-dependent Hamiltonian $H_{0}(t)$ is much slower than the time scales characterizing the reservoir [30].

Formal solution of the master equation and its connection with GPs. Expanding $\rho(t)$ in the eigenstate basis of the invariant, we obtain through the master equation in 
the Lindblad form, the coupled differential equations

$$
\begin{gathered}
c_{\mu, \mu^{\prime}}^{. b, b^{\prime}}=i \sum_{\lambda, a}\left(H_{\mu, \lambda}^{b, a}+A_{\mu, \lambda}^{b, a}+i D_{\mu, \lambda}^{b, a}\right) c_{\lambda, \mu^{\prime}}^{a, b^{\prime}} \\
-i \sum_{\lambda, a} c_{\mu, \lambda}^{b, a}\left(H_{\lambda, \mu^{\prime}}^{a, b^{\prime}}+A_{\lambda, \mu^{\prime}}^{a, b^{\prime}}-i D_{\lambda, \mu^{\prime}}^{a, b^{\prime}}\right) \\
+2 \sum_{i, j} \gamma_{i j} \sum_{\lambda, \lambda^{\prime}, a, a^{\prime}} \Lambda_{\mu, \lambda ; i}^{b, a} c_{\lambda, \lambda^{\prime}}^{a, a^{\prime}}\left(\Lambda_{\mu^{\prime}, \lambda^{\prime} ; j}^{b^{\prime}, a^{\prime}}\right)^{*},
\end{gathered}
$$

where we have defined the time-dependent matrix elements $H_{\mu, \lambda}^{b, a}=-\left\langle\mu, b ; t\left|H_{0}\right| \lambda, a ; t\right\rangle, \quad A_{\mu, \lambda}^{b, a}=$ $\left\langle\mu, b ; t\left|i d_{t}\right| \lambda, a ; t\right\rangle, \quad D_{\mu, \lambda}^{b, a}=\sum_{i, j} \gamma_{i j}\left\langle\mu, b ; t\left|\Gamma_{j}^{\dagger} \Gamma_{i}\right| \lambda, a ; t\right\rangle$, and $\Lambda_{\mu, \lambda ; i}^{b, a}=\left\langle\mu, b ; t\left|\Gamma_{i}\right| \lambda, a ; t\right\rangle$. It can be verified that the formal integration of Eq. (44) leads to

$$
\begin{aligned}
c(t) & =T \exp \left\{i \int_{0}^{t} d \tau[H+A+i D](\cdot)+2 \sum_{i, j} \int_{0}^{t} d \tau\right. \\
& \left.\times \gamma_{i j} \Lambda_{i}(\cdot) \Lambda_{j}^{\dagger}-i(\cdot) \int_{0}^{t} d \tau[H+A-i D]\right\} c(0), \quad(5)
\end{aligned}
$$

with (.) indicating the side where the matrices $H+A \pm i D$, $\Lambda_{i}$ and $\Lambda_{j}^{\dagger}$, are supposed to act on $c(0)$, in the timeordered expansion of the r.h.s. of (5). Differently from the original procedure by Berry [1] (within the context of an adiabatic and cyclic evolution of a pure state), it is no longer clear, as first noted in Ref. [26], how to extract the GP from the dynamics of the probability amplitudes (Eq. (5)), describing the nonadiabatic evolution of an open system, since in general $A$ does not commute with $H, D$, and $\Lambda_{i}$. Some exceptions correspond to the specific cases of open quantum systems discussed in Refs. [15, 17] and filtering evolutions 31]. That is why we have presented a formal definition of the GP in Eq. (2), instead of trying to obtain it, as usual [1, 25, 28, 32], from the dynamics of the probability amplitudes. For some particular situations of dissipative-free dynamics, a connection can be established between the GPs emerging from our definition and the dynamics of the probability amplitudes coefficients, as shown below.

Dissipative-free dynamics (degenerate case). For dissipative-free dynamics $\left[\gamma_{i j}(t)=0\right.$ in Eq. (3) $]$ the solution (5), inside the degenerate subspaces $\mu$ and $\mu^{\prime}$, reduces to

$$
\begin{aligned}
c_{\mu, \mu^{\prime}}(t) & =\left(T \mathrm{e}^{i \int_{0}^{t} d \tau\left(H_{\mu, \mu}+A_{\mu, \mu}\right)}\right) c_{\mu, \mu^{\prime}}(0) \\
& \times T \mathrm{e}^{-i \int_{0}^{t} d \tau\left(H_{\mu^{\prime}, \mu^{\prime}}+A_{\mu^{\prime}, \mu^{\prime}}\right)},
\end{aligned}
$$

where $c_{\mu, \mu^{\prime}}, H_{\mu, \mu}$, and $A_{\mu, \mu}$ represent matrices composed of the elements $c_{\mu, \mu^{\prime}}^{b, a}, H_{\mu, \mu}^{b, a}$, and $A_{\mu, \mu}^{b, a}$. In Ref. [32], the assumption that $\left[H_{\mu, \mu}, A_{\mu, \mu}\right]=0$ even for a nonadiabatic evolution, resulted in the expressions $T \exp \left[i \int_{0}^{t} d \tau H_{\mu, \mu}(\tau)\right]$ for dynamic and $T \exp \left[i \int_{0}^{t} d \tau A_{\mu, \mu}(\tau)\right]$ for cyclic geometric phases. However, as in general $\left[H_{\mu, \mu}, A_{\mu, \mu}\right] \neq 0$ for transitional and nonadiabatic dynamics in a degenerate subspace $\mu$, we must return to the formal expression (2) with $V_{\|}^{\mathrm{t}, \mathrm{d}}(t)=\sum_{\mu} \sum_{a, a^{\prime}}\left(V_{\|}\right)_{\mu \mu}^{a a^{\prime}}(t)|\mu, a ; t\rangle\left\langle\mu, a^{\prime} ; 0\right|$ to obtain the GPs for cyclic $(U=\mathbf{1})$ and noncyclic $(U \neq \mathbf{1})$ evolutions. For the cyclic case, Eq. (2) with $V_{\|}^{\mathrm{t}, \mathrm{d}}(t)$ leads exactly to the expression $T \exp \left[i \int_{0}^{t} d \tau A_{\mu, \mu}(\tau)\right][32]$ for the GP, whereas for the noncyclic case the matrix $U$ must be taken into account (instead of matrix $W$ as in Ref. [28]), with the elements of the non-Abelian connection being given by $A_{\mu, \mu}^{a, a^{\prime}}(\tau)=i\left\langle\mu, a ; \tau\left|d_{\tau}\right| \mu, a^{\prime} ; \tau\right\rangle$.

For transitional and adiabatic dynamics in a degenerate subspace, the adiabatic evolution $V_{\|}^{\mathrm{t}, \mathrm{d}}(t)$ is built up from the condition $\partial_{t} I \simeq 0$ [22], which implies that $H_{0}$ and $I$ assume the same basis $\{|\mu, a ; t\rangle\}$. In this case, the non-Abelian dynamic phase reduces to the Abelian one, since $H_{\mu, \mu}^{b, a}=E_{\mu} \delta_{a b}$, where $E_{\mu}$ is the eigenenergy associated with the state $|\mu, a ; t\rangle$ and, consequently, $\left[H_{\mu, \mu}, A_{\mu, \mu}\right]=0$. The cyclic GP thus remains the nonAbelian holonomy $T \exp \left[i \int_{0}^{t} d \tau A(\tau)\right]$ computed in the Hamiltonian eigenstates, obtained by Wilczek and Zee 25]. For a noncyclic evolution, the nonidentity matrix $U$ is responsible for yielding the result in Ref. [27].

Dissipative-free dynamics (nondegenerate case). For the nondegenerate case, we easily verify that the solution of (44), corresponding to a particular case of Eq. (6), is given by $c_{\mu, \mu^{\prime}}(t)=$ $c_{\mu, \mu^{\prime}}(0) \exp \left\{i \int_{0}^{t} d \tau\left[\Delta H_{\mu, \mu^{\prime}}(\tau)+\Delta A_{\mu, \mu^{\prime}}(\tau)\right]\right\}, \quad$ with $\Delta H_{\mu, \mu^{\prime}}=H_{\mu, \mu}-H_{\mu^{\prime}, \mu^{\prime}}, H_{\mu, \mu}=-\left\langle\mu ; t\left|H_{0}\right| \mu ; t\right\rangle$, $\Delta A_{\mu, \mu^{\prime}}=A_{\mu, \mu}-A_{\mu^{\prime}, \mu^{\prime}}$, and $A_{\mu, \mu}=\left\langle\mu ; t\left|i d_{t}\right| \mu ; t\right\rangle$, so that the dynamic and cyclic GPs reduce to the Abelian expressions $\exp \left[i \int_{0}^{t} d \tau H_{\mu, \mu}(\tau)\right]$ and $\exp \left[i \int_{0}^{t} d \tau A_{\mu, \mu}(\tau)\right]$. Note that the dynamic and geometric phases associated with the element $\rho_{\mu, \mu^{\prime}}$ is simply the difference between the phases acquired by the states $|\mu ; t\rangle$ and $\left|\mu^{\prime} ; t\right\rangle$, even for the nonadiabatic case, as can be verified from the DIs [18]. This striking feature of the DIs enable nonadiabatic evolutions with non-transitional eigenstates in the nondegenerate case. In this connection, the operators $U^{\text {nt,nd }}(t, 0)$ and $V_{\|}^{\mathrm{nt}, \mathrm{nd}}(t)=\sum_{\lambda}\left(V_{\|}\right)_{\lambda \lambda}(t)|\lambda ; t\rangle\langle\lambda ; 0|$ in Eq. (2) describe the GP for noncyclic evolutions whose expression is $\langle\lambda ; 0 \mid \lambda ; t\rangle \exp \left(-\int_{0}^{t} d \tau\left\langle\lambda ; \tau\left|d_{\tau}\right| \lambda ; \tau\right\rangle\right)$ [28]. For cyclic evolutions this GP reduces to that obtained in Refs. 21, 22]. Evidently, for the adiabatic case $\left(\partial_{t} I \simeq 0\right)$ the above expression for the noncyclic GP gives the result in Ref. [33], while in the cyclic case it reproduces the original Berry phase [1].

Application - An unstable two-level system. A particular form of the operator $V_{11}(t)$, given by $\left[V_{11}(t)\right]^{\text {t,nd }}=$ $\sum_{\lambda, \lambda^{\prime}}\left(V_{11}\right)_{\lambda \lambda^{\prime}}(t)|\lambda ; t\rangle\left\langle\lambda^{\prime} ; 0\right|$, occurs when we have a nonadiabatic evolution with a transition between nondegenerate states of the basis $\{|\lambda ; t\rangle\}$. This is the case 
in a quantum system undergoing a unitary evolution, as in Ref. [26], or a nonunitary evolution where, as shown below, the noise injection gives rise to a non-Abelian GP.

Employing the above method to analyze the role of dissipation in the evolution of the GP, we consider a nondegenerate two-level system, with transition frequency $\omega_{0}$ between the ground $(g)$ and excited $(e)$ states, under spontaneous decay at $0 \mathrm{~K}$. The dynamics of this system is described by the solution to the master equation for $\rho(t)$ with $H_{0}=\omega_{0} \sigma_{z} / 2, \gamma_{11}=\gamma / 2$, and $\Gamma_{1}=\sigma_{-}$. For the invariant operator, we assume

$$
I(t)=\sum_{\alpha, \beta=g, e} \chi_{\alpha \beta}(t) \sigma_{\alpha \beta},
$$

where $\sigma_{\alpha \beta}=|\alpha\rangle\langle\beta|$ and the coefficients $\chi_{\alpha \beta}(t)$, which are solutions of Eq. (3), satisfy the relations

$$
\begin{aligned}
& \chi_{g g}(t)=-r_{0} \cos \theta_{0}, \\
& \chi_{e e}(t)=\left(2 \mathrm{e}^{\gamma t}-1\right) r_{0} \cos \theta_{0}, \\
& \chi_{e g}(t)=r_{0} \sin \theta_{0} \mathrm{e}^{\gamma t / 2-i\left(\omega_{0} t+\phi_{0}\right)}, \\
& \chi_{g e}(t)=r_{0} \sin \theta_{0} \mathrm{e}^{\gamma t / 2+i\left(\omega_{0} t+\phi_{0}\right)},
\end{aligned}
$$

with $\theta_{0} \quad\left(\phi_{0}\right)$ being the polar (azimuthal) angle of the initial pure $\left(r_{0}=1\right)$ or mixed $\left(r_{0}<1\right)$ state $\rho(0)$ in the Bloch sphere. The eigenvalues and eigenvectors of the invariant (77) are given by $\lambda_{ \pm}(t)=$ $-r_{0}\left[\left(1-\mathrm{e}^{\gamma t}\right) \cos \theta_{0} \mp \mathrm{e}^{\gamma t / 2} \sqrt{1-\left(1-\mathrm{e}^{\gamma t}\right) \cos ^{2} \theta_{0}}\right]$, $| \pm ; t\rangle= \pm N(t)\left\{f(t)\left|\begin{array}{l}g \\ e\end{array}\right\rangle \pm r_{0} \sin \theta_{0} \mathrm{e}^{\mp i\left(\omega_{0} t+\phi_{0}\right)}\left|\begin{array}{l}e \\ g\end{array}\right\rangle\right\}$, with $f(t)=-r_{0}\left[\mathrm{e}^{\gamma t / 2} \cos \theta_{0}-\sqrt{1-\left(1-\mathrm{e}^{\gamma t}\right) \cos ^{2} \theta_{0}}\right]$ and $N^{2}(t)=\left(f^{2}(t)+r_{0}^{2} \sin ^{2} \theta_{0}\right)^{-1}$.

In order to find the coefficients $\left(V_{11}\right)_{\lambda \lambda^{\prime}}(t)$ of $\left[V_{11}(t)\right]^{\mathrm{t}, \mathrm{nd}}$ we first solve the equation $\left[\dot{V}_{\|}(t)\right]^{\mathrm{t}, \mathrm{nd}}=i A(t)\left[V_{\Perp}(t)\right]^{\mathrm{t}, \mathrm{nd}}$, where the elements of the non-Abelian connection are given by $A_{k l}=i\left\langle k ; t\left|d_{t}\right| l ; t\right\rangle$, with $k, l=+,-$. To this end, we move to the rotating frame $R=$ $\exp \left[\eta\left(\mathrm{e}^{-i \zeta} \sigma_{-}-\mathrm{e}^{i \zeta} \sigma_{+}\right) / 2\right]$, as in Ref. [20], obtaining the coupled differential equations

$$
\begin{aligned}
\dot{\eta} & =2 N^{2} r_{0} \sin \theta_{0}\left[\omega_{0} f \sin \Theta+\dot{f} \cos \Theta\right], \\
\dot{\zeta} & =2 N^{2} r_{0} \sin \theta_{0}\left\{\omega_{0} r_{0} \sin \theta_{0}\right. \\
& \left.+\cot \eta\left[-\omega_{0} f \cos \Theta+\dot{f} \sin \Theta\right]\right\},
\end{aligned}
$$

where $\Theta=\omega_{0} t+\phi_{0}-\zeta$. Under the initial condition $\pi / 7 \lesssim \theta_{0} \lesssim \pi$ (which is necessary to obtain an approximated analytical solution), the assumption of a typical weak system-reservoir coupling, $\gamma / \omega_{0} \ll 1$, and assuming a time evolution around $t \simeq 2 \pi / \omega_{0}$, we obtain $\dot{\eta} \simeq 0$, such that $\eta(t) \simeq \operatorname{arccot}\left\{\cot \theta_{0}\left[1+\frac{\left(1-\cos \theta_{0} / 2\right)}{1-\cos \theta_{0}} \gamma t\right]\right\}$ and $\zeta(t)=\omega_{0} t+\phi_{0}-\gamma \cos \theta_{0} / 2 \omega_{0}$.

¿From the above result, we obtain the noncyclic, nonadiabatic, and non-Abelian GP associated with the decaying two-level system $\mathcal{O}^{\mathrm{t}, \mathrm{nd}}(t, 0)=U(t, 0) R^{\dagger}(t) \mathrm{e}^{i \Omega(t) \sigma_{z}} R(0)$, where $\Omega(t) \simeq \omega_{0} t\left[1+\gamma S\left(\theta_{0}\right) t / 2\right], \quad S\left(\theta_{0}\right)=$ $-\cos \theta_{0}\left(1 / 2-\cos \theta_{0}+3 \cos ^{2} \theta_{0} / 8\right) /\left(1-\cos \theta_{0}\right)$, and

$$
U(t, 0)=\left(\begin{array}{cc}
U_{D} & -U_{O D}^{*} \\
U_{O D} & U_{D}^{*}
\end{array}\right)
$$

with the on- and off-diagonal elements of the overlap matrix given, respectively, by $U_{D}=N \sin \left(\theta_{0} / 2\right)\left[f+2 r_{0} \mathrm{e}^{-i \omega_{0} t} \cos ^{2}\left(\theta_{0} / 2\right)\right]$ and $U_{O D}=N \mathrm{e}^{-i \phi_{0}} \cos \left(\theta_{0} / 2\right)\left[f-2 r_{0} \mathrm{e}^{-i \omega_{0} t} \sin ^{2}\left(\theta_{0} / 2\right)\right]$. At this point, we stress that the decay process introduced by the reservoir leads to a transitional dynamics of the DI basis states, bringing about a not fault-tolerant non-Abelian GP. In the particular case $\gamma=0$ (nondegenerate dissipative-free dynamics), the non-transitional evolution during the time interval $2 \pi / \omega_{0}$ leads to the cyclic GPs $\mathcal{O}_{ \pm}^{\text {nt,nd }}\left(2 \pi / \omega_{0}, 0\right)= \pm \pi\left(1-\cos \theta_{0}\right)$ associated with the eigenstates $| \pm ; t\rangle$, as obtained in Refs. $([1,22])$.

Whereas some works are concerned with a formal definition of the GPs for open quantum systems [12, 13, 14, 15, 16, 17], most of them restrict themselves to compute corrections to this phase coming from the reservoir 8, 9, 10, 11]. From the formal approach presented in this work we additionally verify that the reservoir may even change the nature of the holonomy, from an Abelian $(\gamma=0)$ to a non-Abelian $(\gamma \neq 0)$ one, as it emerges from the above application.

Summarizing, we have presented a general formalism to compute GPs, starting only from the parallel transport condition. These GPs transform covariantly and the approach is applicable to a general scenario, including adiabatic or nonadiabatic, cyclic or noncyclic, and transitional or non-transitional evolutions of pure or mixed states. Although we have used the DIs to compute the GPs acquired by the basis states of the invariant, the formalism is applicable to any time-dependent basis states. Besides reproducing well-known results established in the literature, our formal definition reveals two striking features of the GP: it generalizes Wilczek and Zee's non-Abelian holonomy [25], in describing nonadiabatic evolutions where the basis states are parallelly transported between distinct degenerate subspaces; secondly, our method demonstrates clearly that the non-Abelian character of the GP arises from transitional dynamics, even in nondegenerate case. We have shown that the nonadiabatic evolution of an open two-level quantum system introduces a non-Abelian holonomy. Both of these features not only deepen our understanding of GPs, but also offer the possibility of investigating how to use the non-Abelian holonomy acquired by transitional dynamics between nondegenerate states to perform geometric quantum computation.

We gratefully acknowledge financial support from the Brazilian agencies CNPq and UFABC (to E.I.D.), and 
CNPq and FAPESP (to R.M.S. and M.H.Y.M.). We are also grateful to an anonymous referee for valuable comments.

* Electronic address: duzzioni@ufabc.edu.br

† Electronic address: serra@ufabc.edu.br

‡ Electronic address: miled@ifsc.usp.br

[1] M. V. Berry, Proc. Roy. Soc. London A 392, 45 (1984).

[2] S. Pancharatnam, Proc. Ind. Acad. Sci. A44, 247 (1956).

[3] Y. Aharonov and J. Anandan, Phys. Rev. Lett. 58, 1593 (1987).

[4] J. Samuel and R. Bhandari, Phys. Rev. Lett. 60, 2339 (1988).

[5] P. Zanardi and M. Rasetti, Phys. Lett. A 264, 94 (1999).

[6] J. Pachos, et al., Phys. Rev. A 61, 010305(R) (1999); A. Recati, et al., ibid. 66, 032309 (2002); P. Solinas, et al., ibid. 67, 052309 (2003); Li-X. Cen and P. Zanardi, ibid. 70, 052323 (2004); A. Ekert, et al., J. Mod. Opt. 47, 2501 (2000); L. M. Duan, et al., Science 292, 1695 (2001); L. Faoro, J. Siewert, and R. Fazio, Phys. Rev. Lett. 90, 028301 (2003); I. Fuentes-Guridi, et al., ibid. 94, 020503 (2005).

[7] A. Carollo and V. Vedral, quant-ph/0504205 (2005).

[8] J. C. Garrison and E. M. Wright, Phys. Lett. A 128, 177 (1988); G. Dattoli, R. Mignani, and A. Torre, J. Phys. A 23, 5795 (1990).

[9] G. De Chiara and G. M. Palma, Phys. Rev. Lett. 91, 090404 (2003); A. Blais and A. -M. S. Tremblay, Phys. Rev. A 67, 012308 (2003).

[10] D. Ellinas, S. M. Barnett, and M. A. Dupertuis, Phys. Rev. A 39, 3228 (1989); D. Gamliel and J. H. Freed, Phys. Rev. A 39, 3238 (1989); K. M. F. Romero, A. C. A. Pinto, and M. T. Thomaz, Physica A 307, 142 (2002); A. C. A. Pinto and M. T. Thomaz, J. Phys. A 36, 7461 (2003); R. S. Whitney and Y. Gefen, Phys. Rev. Lett. 90, 190402 (2003); I. Kamleitner, J. D. Cresser, and B. C. Sanders, Phys. Rev. A 70, 044103 (2004); R. S. Whitney, Y. Makhlin, A. Shnirman, and Y. Gefen, Phys. Rev. Lett. 94, 070407 (2005); F. C. Lombardo and P. I.
Villar, Phys. Rev. A 74, 042311 (2006).

[11] A. Nazir, T. P. Spiller, and W. J. Munro, Phys. Rev. A 65, 042303 (2002); A. Carollo, et al., Phys. Rev. Lett. 90, 160402 (2003); A. Bassi and E. Ippoliti, Phys. Rev. A 73, 062104 (2006).

[12] D. M. Tong, et al., Phys. Rev. Lett. 93, 080405 (2004); D. M. Tong, et al., ibid. 95, 249902(E) (2005); A. T. Rezakhani and P. Zanardi, Phys. Rev. A 73, 012107 (2006).

[13] K. -P. Marzlin, et al., Phys. Rev. Lett. 93, 260402 (2004).

[14] J. G. P. de Faria, A. F. R. de Toledo Piza, and M. C. Nemes, Europhys. Lett. 62, 782 (2003); M. Ericsson, et al., Phys. Rev. A 67, 020101(R) (2003).

[15] M. S. Sarandy and D. A. Lidar, Phys. Rev. A 73, 062101 (2006).

[16] H. Goto and K. Ichimura, Phys. Rev. A 76, 012120 (2007).

[17] M. S. Sarandy, E. I. Duzzioni, and M. H. Y. Moussa, Phys. Rev. A 76, 052112 (2007).

[18] H. R. Lewis and W. B. Riesenfeld, J. Math. Phys. 10, 1458 (1969).

[19] C. J. Villas-Boas, et al., Phys. Rev. 68, 053808 (2003).

[20] E. I. Duzzioni, et al., Phys. Rev. A 75, 032113 (2007).

[21] D. A. Morales, J. Phys. A: Math. Gen. 21, L889 (1988).

[22] S. S. Mizrahi, Phys. Lett. A 138, 465 (1989).

[23] E. I. Duzzioni, et al., Europhysics Letters 72, 21 (2005).

[24] V. V. Dodonov and V. I. Man'ko, Physica A 94, 403 (1978).

[25] F. Wilczek and A. Zee, Phys. Rev. Lett. 52, 2111 (1984).

[26] J. Anandan, Phys. Lett. A 133, 171 (1988).

[27] D. Kult, et al., Phys. Rev. 74, 022106 (2006).

[28] A. Mostafazadeh, J. Phys. A: Math. Gen. 32, 8157 (1999).

[29] N. Manini and F. Pistolesi, Phys. Rev. Lett. 85, 3067 (2000).

[30] E. B. Davies and H. Spohn, J. Stat. Phys. 19, 511 (1978); G. Florio, et al., Phys. Rev. A 73, 022327 (2006).

[31] D. Kult, J. Aberg, and E. Sjöqvist, Europhys. Lett. 78, 60004 (2007).

[32] A. Mostafazadeh, J. Phys. A: Math. Gen. 31, 9975 (1998).

[33] G. G. de Polavieja and E. Sjöqvist, Am. J. Phys. 66, 431 (1998). 